\title{
Formation of the $\eta_{c}$ in two-photon collisions at LEP
}

\section{L3 Collaboration}

M. Acciarri y, P. Achard ${ }^{\text {r }}$, O. Adriani ${ }^{\circ}$, M. Aguilar-Benitez x , J. Alcaraz ${ }^{\text {x }}$, G. Alemanni ", J. Allaby ${ }^{\mathrm{p}}$, A. Aloisio ${ }^{\text {aa }}$, M.G. Alviggi ${ }^{\text {aa }}$, G. Ambrosi ${ }^{\mathrm{r}}$, H. Anderhub at, V.P. Andreev f,ai , T. Angelescu ' , F. Anselmo i, A. Arefiev ${ }^{\text {z }}$, T. Azemoon ${ }^{\text {c }}$, T. Aziz ${ }^{\mathrm{j}}$, P. Bagnaia ${ }^{\text {ah }}$, L. Baksay $^{\text {ao }}$, A. Balandras ${ }^{\mathrm{d}}$, R.C. Ball ${ }^{\mathrm{c}}$, S. Banerjee ${ }^{j}$, Sw. Banerjee ${ }^{j}$, A. Barczyk ${ }^{\text {at,ar }}$, R. Barillère ${ }^{p}$, L. Barone $^{\text {ah }}$, P. Bartalini " , M. Basile i, R. Battiston ${ }^{\text {ae }}$, A. Bay ${ }^{\text {u }}$, F. Becattini ${ }^{\circ}$, U. Becker ${ }^{n}$, F. Behner ${ }^{\text {at }}$, J. Berdugo ${ }^{\mathrm{x}}$, P. Berges ${ }^{\mathrm{n}}$, B. Bertucci ${ }^{\text {ae }}$, B.L. Betev ${ }^{\text {at }}$, S. Bhattacharya ${ }^{j}$, M. Biasini ${ }^{\text {ae }}$, A. Biland ${ }^{\text {at }}$, J.J. Blaising ${ }^{\text {d }}$, S.C. Blyth ${ }^{\text {af }}$, G.J. Bobbink ${ }^{\text {b }, ~ A . ~ B o ̈ h m ~ a ~, ~ L . ~ B o l d i z s a r ~ m, ~ B . ~ B o r g i a ~}{ }^{\text {ah }}$, D. Bourilkov at, M. Bourquin ${ }^{\mathrm{r}}$, S. Braccini ${ }^{\mathrm{r}}$, J.G. Branson ${ }^{\text {ak }}$, V. Brigljevic ${ }^{\text {at }}$, F. Brochu ${ }^{\mathrm{d}}$, A. Buffini ${ }^{\circ}$, A. Buijs ${ }^{\text {ap }}$, J.D. Burger ${ }^{n}$, W.J. Burger ${ }^{\text {ae }}$, J. Busenitz ${ }^{\text {ao }}$, A. Button ${ }^{\text {c }}$,

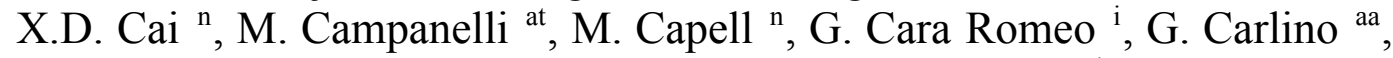
A.M. Cartacci ${ }^{\circ}$, J. Casaus ${ }^{\mathrm{x}}$, G. Castellini ${ }^{\circ}$, F. Cavallari ah, N. Cavallo ${ }^{\text {aa }}$, C. Cecchi ${ }^{\text {r }}$, M. Cerrada ${ }^{\text {x }}$, F. Cesaroni ${ }^{\mathrm{v}}$, M. Chamizo ${ }^{\mathrm{r}}$, Y.H. Chang ${ }^{\text {av }}$, U.K. Chaturvedi ${ }^{\mathrm{q}}$, M. Chemarin ${ }^{\mathrm{w}}$, A. Chen ${ }^{\text {av }}$, G. Chen ${ }^{\mathrm{g}}$, G.M. Chen ${ }^{\mathrm{g}}$, H.F. Chen ${ }^{\mathrm{s}}$, H.S. Chen ${ }^{\mathrm{g}}$, X. Chereau ${ }^{\mathrm{d}}$, G. Chiefari ${ }^{\text {aa }}$, L. Cifarelli aj, F. Cindolo ${ }^{\mathrm{i}}$,

C. Civinini ${ }^{\circ}$, I. Clare ${ }^{n}$, R. Clare ${ }^{n}$, G. Coignet ${ }^{d}$, A.P. Colijn ${ }^{b}$, N. Colino ${ }^{x}$, S. Costantini ${ }^{\text {h }}$, F. Cotorobai ${ }^{1}$, B. Cozzoni i , B. de la Cruz ${ }^{\mathrm{x}}$, A. Csilling ${ }^{\mathrm{m}}$, S. Cucciarelli ${ }^{\text {ae }}$, T.S. Dai ${ }^{\text {n }}$, J.A. van Dalen ${ }^{\text {ac }}$, R. D’Alessandro ${ }^{\circ}$, R. de Asmundis ${ }^{\text {aa }}$,

P. Deglon ${ }^{\mathrm{r}}$, A. Degré ${ }^{\mathrm{d}}$, K. Deiters ${ }^{\text {ar }}$, D. della Volpe ${ }^{\text {aa }}$, P. Denes ${ }^{\text {ag }}$, F. DeNotaristefani ${ }^{\text {ah }}$, A. De Salvo ${ }^{\text {at }}$, M. Diemoz ah ${ }^{\text {a }}$ D. van Dierendonck ${ }^{\text {b }}$, F. Di Lodovico at, C. Dionisi ${ }^{\text {ah }}$, M. Dittmar ${ }^{\text {at }}$, A. Dominguez ${ }^{\text {ak }}$, A. Doria ${ }^{\text {aa }}$, M.T. Dova ${ }^{\text {q,1 }}$, D. Duchesneau d, D. Dufournand ${ }^{\text {d }}$, P. Duinker ${ }^{\text {b }}$, I. Duran ${ }^{\text {al }}$, H. El Mamouni ${ }^{\text {w }}$, A. Engler af , F.J. Eppling ${ }^{\text {n }}$, F.C. Erné ${ }^{\text {b }}$, P. Extermann ${ }^{\text {r }}$, M. Fabre ${ }^{\text {ar }}$, R. Faccini ah, M.A. Falagan ${ }^{x}$, S. Falciano ${ }^{\text {ah, }}$, A. Favara ${ }^{\text {p }}$, J. Fay ${ }^{\text {w }}$, O. Fedin ${ }^{\text {ai }}$, M. Felcini at, T. Ferguson ${ }^{\text {af }}$, F. Ferroni ${ }^{\text {ah }}$, H. Fesefeldt ${ }^{a}$, E. Fiandrini ${ }^{\text {ae }}$, J.H. Field $^{\mathrm{r}}$, F. Filthaut ${ }^{\mathrm{p}}$, P.H. Fisher ${ }^{\mathrm{n}}$, I. Fisk ${ }^{\text {ak }}$, G. Forconi ${ }^{\mathrm{n}}$, L. Fredj ${ }^{\mathrm{r}}$, K. Freudenreich ${ }^{\text {at }}$, C. Furetta ${ }^{y}$, Yu. Galaktionov ${ }^{z, n}$, S.N. Ganguli ${ }^{j}$, P. Garcia-Abia e, 
M. Gataullin ${ }^{\text {ad }}$, S.S. Gau ${ }^{\text {k }}$, S. Gentile ${ }^{\text {ah,p }}$, N. Gheordanescu ${ }^{1}$, S. Giagu ${ }^{\text {ah }}$, Z.F. Gong s, G. Grenier ${ }^{\text {w }}$, O. Grimm ${ }^{\text {at }}$, M.W. Gruenewald ${ }^{\text {h }}$, R. van Gulik ${ }^{\mathrm{b}}$, V.K. Gupta ${ }^{\text {ag }}$, A. Gurtu j, L.J. Gutay ${ }^{\text {aq }}$, D. Haas ${ }^{\text {e }}$, A. Hasan ${ }^{\text {ab }}$, D. Hatzifotiadou ${ }^{\text {i }}$, T. Hebbeker ${ }^{\text {h }}$, A. Hervé ${ }^{\mathrm{p}}$, P. Hidas ${ }^{\mathrm{m}}$, J. Hirschfelder ${ }^{\text {af }}, \mathrm{H}$. Hofer ${ }^{\text {at }}$, G. Holzner ${ }^{\text {at }}$, H. Hoorani ${ }^{\text {af }}$, S.R. Hou ${ }^{\text {av }}$, I. Iashvili as, B.N. Jin ${ }^{\text {g }}$, L.W. Jones ${ }^{\text {c }}$, P. de Jong ${ }^{\mathrm{b}}$,

I. Josa-Mutuberría ${ }^{x}$, R.A. Khan ${ }^{\mathrm{q}}$, D. Kamrad ${ }^{\text {as }}$, M. Kaur ${ }^{\mathrm{q}, 2}$,

M.N. Kienzle-Focacci ${ }^{\text {r }}$, D. Kim ${ }^{\text {ah }}$, D.H. Kim ${ }^{\text {an }}$, J.K. Kim ${ }^{\text {an }}$, S.C. Kim ${ }^{\text {an }}$, J. Kirkby ${ }^{\text {p }}$, D. Kiss ${ }^{\mathrm{m}}$, W. Kittel ${ }^{\text {ac }}$, A. Klimentov ${ }^{\text {n,z }}$, A.C. König ${ }^{\text {ac }}$, A. Kopp ${ }^{\text {as }}$, I. Korolko ${ }^{\text {, }}$, V. Koutsenko ${ }^{\text {n,z }}$, M. Kräber ${ }^{\text {at }}$, R.W. Kraemer af , W. Krenz a , A. Kunin ${ }^{n, z}$, P. Lacentre ${ }^{\text {as, } 1,3}$, P. Ladron de Guevara ${ }^{x}$, I. Laktineh ${ }^{\text {w }}$, G. Landi ${ }^{\circ}$, K. Lassila-Perini ${ }^{\text {at }}$, P. Laurikainen ${ }^{t}$, A. Lavorato ${ }^{\text {aj }}$, M. Lebeau ${ }^{p}$, A. Lebedev ${ }^{n}$, P. Lebrun ${ }^{\text {w }}$, P. Lecomte ${ }^{\text {at }}$, P. Lecoq ${ }^{\text {p }}$, P. Le Coultre ${ }^{\text {at }}$, H.J. Lee ${ }^{\text {h }}$, J.M. Le Goff ${ }^{p}$, R. Leiste ${ }^{\text {as }}$, E. Leonardi ah, P. Levtchenko ai, C. Li ${ }^{\text {s }}$, C.H. Lin ${ }^{\text {av }}$, W.T. Lin ${ }^{\text {av }}$, F.L. Linde ${ }^{b}$, L. Lista ${ }^{\text {aa }}$, Z.A. Liu ${ }^{\mathrm{g}}$, W. Lohmann ${ }^{\text {as }}$, E. Longo ${ }^{\text {ah }}$, Y.S. Lu ${ }^{\mathrm{g}}$, K. Lübelsmeyer ${ }^{\text {a }}$, C. Luci ${ }^{\text {p,ah }}$, D. Luckey ${ }^{n}$, L. Lugnier ${ }^{\text {w }}$, L. Luminari ${ }^{\text {ah }}$, W. Lustermann ${ }^{\text {at }}$, W.G. Ma ${ }^{\text {s }}$, M. Maity ${ }^{j}$, L. Malgeri ${ }^{\mathrm{p}}$, A. Malinin ${ }^{\text {z,p }}$, C. Maña ${ }^{\mathrm{x}}$, D. Mangeol ac, P. Marchesini ${ }^{\text {at }}$, G. Marian ${ }^{\text {ao, } 4}$, J.P. Martin ${ }^{\text {w }}$, F. Marzano ${ }^{\text {ah }}$, G.G.G. Massaro ${ }^{\text {b }}$, K. Mazumdar ${ }^{\text {j }}$, R.R. McNeil ${ }^{\mathrm{f}}$, S. Mele ${ }^{\mathrm{p}}$, L. Merola ${ }^{\text {aa }}$, M. Meschini ${ }^{\circ}$, W.J. Metzger ${ }^{\text {ac }}$, M. von der Mey ${ }^{\text {a }}$, D. Migani ${ }^{\text {i }}$, A. Mihul ${ }^{1}$, H. Milcent ${ }^{\mathrm{p}}$, G. Mirabelli ah ${ }^{\text {, J. Mnich }}{ }^{\mathrm{p}}$, G.B. Mohanty ${ }^{\mathrm{j}}$, P. Molnar ${ }^{\mathrm{h}}$, B. Monteleoni ${ }^{\circ}$, T. Moulik ${ }^{\text {j, G.S. Muanza }}{ }^{\text {w }}$, F. Muheim ${ }^{\mathrm{r}}$, A.J.M. Muijs ${ }^{\mathrm{b}}$, M. Napolitano ${ }^{\text {aa }}$, F. Nessi-Tedaldi ${ }^{\text {at }}$, H. Newman ${ }^{\text {ad }}$, T. Niessen ${ }^{\text {a }}$, A. Nisati ${ }^{\text {ah }}$, H. Nowak ${ }^{\text {as }}$, Y.D. Oh ${ }^{\text {an }}$, G. Organtini ah, R. Ostonen ${ }^{\mathrm{t}}$, C. Palomares ${ }^{\mathrm{x}}$, D. Pandoulas a , S. Paoletti ah,p, P. Paolucci aa , H.K. Park ${ }^{\text {af }}$, I.H. Park ${ }^{\text {an }}$, G. Pascale ${ }^{\text {ah }}$, G. Passaleva $^{\text {p }, ~ S . ~ P a t r i c e l l i ~ a a ~}{ }^{\text {a }}$ T. Paul ${ }^{k}$, M. Pauluzzi ${ }^{\text {ae }}$, C. Paus ${ }^{\text {p }}$, F. Pauss ${ }^{\text {at }}$, D. Peach $^{\mathrm{p}}$, M. Pedace ${ }^{\text {ah }}$, Y.J. Pei a , S. Pensotti ${ }^{\mathrm{y}}$, D. Perret-Gallix ${ }^{\mathrm{d}}$, B. Petersen ${ }^{\mathrm{ac}}$, D. Piccolo ${ }^{\text {aa }}$, M. Pieri ${ }^{\circ}$, P.A. Piroué ${ }^{\mathrm{ag}}$, E. Pistolesi ${ }^{\mathrm{y}}$, V. Plyaskin ${ }^{\mathrm{z}}$, M. Pohl ${ }^{\text {at }}$, V. Pojidaev ${ }^{z, o}$, H. Postema ${ }^{n}$, J. Pothier ${ }^{p}$, N. Produit ${ }^{\mathrm{r}}$, D.O. Prokofiev ${ }^{\mathrm{aq}}$, D. Prokofiev ai, J. Quartieri aj, G. Rahal-Callot at, ${ }^{\text {a }}$, M.A. Rahaman ${ }^{j}$, N. Raja ${ }^{j}$, R. Ramelli ${ }^{\text {at }}$, P.G. Rancoita ${ }^{\mathrm{y}}$, G. Raven ${ }^{\mathrm{ak}}$, P. Razis ${ }^{\mathrm{ab}}$, D. Ren ${ }^{\text {at }}, \mathrm{M}$. Rescigno ${ }^{\text {ah }}$, S. Reucroft ${ }^{k}$, T. van Rhee ${ }^{\text {ap }}$, S. Riemann ${ }^{\text {as }}$, K. Riles ${ }^{\text {c }}$, A. Robohm ${ }^{\text {at }}$, J. Rodin ${ }^{\text {ao }}$, B.P. Roe ${ }^{\mathrm{c}}$, L. Romero ${ }^{\mathrm{x}}$, A. Rosca ${ }^{\mathrm{h}}, \mathrm{S}$. Rosier-Lees ${ }^{\mathrm{d}}$, J.A. Rubio ${ }^{\mathrm{p}}$, D. Ruschmeier ${ }^{\text {h }}$, H. Rykaczewski ${ }^{\text {at }}$, S. Sarkar $^{\text {ah }}$, J. Salicio ${ }^{\text {p }, ~ E . ~ S a n c h e z ~}{ }^{p}$, M.P. Sanders ${ }^{\text {ac }}$, M.E. Sarakinos ${ }^{\mathrm{t}}$, C. Schäfer ${ }^{\mathrm{a}}$, V. Schegelsky ${ }^{\text {ai }}$, S. Schmidt-Kaerst a , D. Schmitz ${ }^{\text {a }}$, H. Schopper ${ }^{\text {au }}$, D.J. Schotanus ${ }^{\text {ac }}$, J. Schwenke a G. Schwering a , C. Sciacca aa, D. Sciarrino ${ }^{\text {r }}$, A. Seganti i, L. Servoli ae, S. Shevchenko ${ }^{\text {ad }}$, N. Shivarov ${ }^{\text {am }}$, V. Shoutko ${ }^{\text {, }}$ E. Shumilov ${ }^{z}$, A. Shvorob ${ }^{\text {ad }}$, T. Siedenburg ${ }^{\text {a }}$, D. Son ${ }^{\text {an }}$, B. Smith ${ }^{\text {af }}$, P. Spillantini ${ }^{\circ}$, M. Steuer ${ }^{\text {n, }}$ 
D.P. Stickland ${ }^{\mathrm{ag}}$, A. Stone ${ }^{\mathrm{f}}$, H. Stone ${ }^{\mathrm{ag}}$, B. Stoyanov ${ }^{\mathrm{am}}$, A. Straessner ${ }^{\mathrm{a}}$, K. Sudhakar ${ }^{\text {j, G. Sultanov }}{ }^{\text {q }}$, L.Z. Sun ${ }^{\text {s }}$, H. Suter ${ }^{\text {at }}$, J.D. Swain ${ }^{\mathrm{q}}$, Z. Szillasi ${ }^{\text {ao,4 }}{ }^{\text {, }}$ X.W. Tang ${ }^{\mathrm{g}}$, L. Tauscher ${ }^{\mathrm{e}}$, L. Taylor ${ }^{\mathrm{k}}$, C. Timmermans ${ }^{\mathrm{ac}}$, Samuel C.C. Ting ${ }^{\mathrm{n}}$, S.M. Ting ${ }^{\mathrm{n}}$, S.C. Tonwar ${ }^{\mathrm{j}}$, J. Tóth ${ }^{\mathrm{m}}$, C. Tully ${ }^{\mathrm{ag}}$, K.L. Tung ${ }^{\mathrm{g}}$, Y. Uchida ${ }^{\mathrm{n}}$, J. Ulbricht ${ }^{\text {at }}$, E. Valente ${ }^{\text {ah }}$, G. Vesztergombi ${ }^{\mathrm{m}}$, I. Vetlitsky ${ }^{\mathrm{z}}$, D. Vicinanza ${ }^{\text {aj }}$, G. Viertel ${ }^{\text {at }}$, S. Villa ${ }^{\mathrm{k}}$, M. Vivargent ${ }^{\mathrm{d}}$, S. Vlachos ${ }^{\mathrm{e}}$, I. Vodopianov ${ }^{\text {ai }}$, H. Vogel ${ }^{\text {af }}$, H. Vogt ${ }^{\text {as }}$, I. Vorobiev ${ }^{\text {z }}$, A.A. Vorobyov ${ }^{\text {ai }}$, A. Vorvolakos ${ }^{a b}$, M. Wadhwa ${ }^{\text {e }}$, W. Wallraff a , M. Wang ${ }^{\mathrm{n}}$, X.L. Wang ${ }^{\text {s, }}$ Z.M. Wang ${ }^{\mathrm{s}}$, A. Weber ${ }^{\mathrm{a}}$, M. Weber ${ }^{\text {a }}$, P. Wienemann ${ }^{\text {a }}$, H. Wilkens ${ }^{\text {ac }}$, S.X. Wu ${ }^{\text {n }}$, S. Wynhoff ${ }^{\text {a }}$, L. Xia ${ }^{\text {ad }}$, Z.Z. Xu ${ }^{\text {s, B.Z. Yang }}{ }^{\text {s, C.G. Yang }}{ }^{\mathrm{g}}$, H.J. Yang ${ }^{\mathrm{g}}$, M. Yang ${ }^{\mathrm{g}}$, J.B. Ye ${ }^{\mathrm{s}}$, S.C. Yeh ${ }^{\text {aw }}$, J.M. You ${ }^{\text {af }}$, An. Zalite ${ }^{\text {ai }}$, Yu. Zalite ${ }^{\text {ai }}$, Z.P. Zhang ${ }^{\text {s }}$, G.Y. Zhu ${ }^{\mathrm{g}}$, R.Y. Zhu ${ }^{\text {ad }}$, A. Zichichi ${ }^{\text {i,p,q }}$, F. Ziegler ${ }^{\text {as }}$, G. Zilizi ${ }^{\text {ao,4 }}$, M. Zöller ${ }^{\text {a }}$

${ }^{a}$ I. Physikalisches Institut, RWTH, D-52056 Aachen, Germany, and III. Physikalisches Institut, RWTH, D-52056 Aachen, Germany 5

${ }^{\mathrm{b}}$ National Institute for High Energy Physics, NIKHEF, and University of Amsterdam, NL-1009 DB Amsterdam, The Netherlands ${ }^{\mathrm{c}}$ University of Michigan, Ann Arbor, MI 48109, USA

${ }^{\mathrm{d}}$ Laboratoire d'Annecy-le-Vieux de Physique des Particules, LAPP, IN2P3-CNRS, BP 110, F-74941 Annecy-le-Vieux CEDEX, France

e Institute of Physics, University of Basel, CH-4056 Basel, Switzerland

${ }^{\mathrm{f}}$ Louisiana State University, Baton Rouge, LA 70803, USA

${ }^{\mathrm{g}}$ Institute of High Energy Physics, IHEP, 100039 Beijing, China ${ }^{6}$

${ }^{\mathrm{h}}$ Humboldt University, D-10099 Berlin, Germany ${ }^{5}$

${ }^{\mathrm{i}}$ University of Bologna and INFN-Sezione di Bologna, I-40126 Bologna, Italy

${ }^{\mathrm{j}}$ Tata Institute of Fundamental Research, Bombay 400 005, India

${ }^{\mathrm{k}}$ Northeastern University, Boston, MA 02115, USA

${ }^{1}$ Institute of Atomic Physics and University of Bucharest, R-76900 Bucharest, Romania

${ }^{\mathrm{m}}$ Central Research Institute for Physics of the Hungarian Academy of Sciences, H-1525 Budapest 114, Hungary ${ }^{7}$

${ }^{\mathrm{n}}$ Massachusetts Institute of Technology, Cambridge, MA 02139, USA

${ }^{\circ}$ INFN Sezione di Firenze and University of Florence, I-50125 Florence, Italy

${ }^{\mathrm{p}}$ European Laboratory for Particle Physics, CERN, CH-1211 Geneva 23, Switzerland

${ }^{\mathrm{q}}$ World Laboratory, FBLJA Project, CH-1211 Geneva 23, Switzerland

${ }^{\mathrm{r}}$ University of Geneva, CH-1211 Geneva 4, Switzerland

${ }^{s}$ Chinese University of Science and Technology, USTC, Hefei, Anhui 230 029, China ${ }^{6}$

${ }^{\mathrm{t}}$ SEFT, Research Institute for High Energy Physics, P.O. Box 9, SF-00014 Helsinki, Finland

"University of Lausanne, CH-1015 Lausanne, Switzerland

${ }^{\vee}$ INFN-Sezione di Lecce and Universitá Degli Studi di Lecce, I-73100 Lecce, Italy

${ }^{\mathrm{w}}$ Institut de Physique Nucléaire de Lyon, IN2P3-CNRS, Université Claude Bernard, F-69622 Villeurbanne, France

${ }^{x}$ Centro de Investigaciones Energéticas, Medioambientales y Tecnologícas, CIEMAT, E-28040 Madrid, Spain ${ }^{8}$

${ }^{\mathrm{y}}$ INFN-Sezione di Milano, I-20133 Milan, Italy

${ }^{\mathrm{z}}$ Institute of Theoretical and Experimental Physics, ITEP, Moscow, Russia

aa INFN-Sezione di Napoli and University of Naples, I-80125 Naples, Italy

ab Department of Natural Sciences, University of Cyprus, Nicosia, Cyprus

ac University of Nijmegen and NIKHEF, NL-6525 ED Nijmegen, The Netherlands

ad California Institute of Technology, Pasadena, CA 91125, USA

ae INFN-Sezione di Perugia and Universitá Degli Studi di Perugia, I-06100 Perugia, Italy

af Carnegie Mellon University, Pittsburgh, PA 15213, USA

ag Princeton University, Princeton, NJ 08544, USA

ah INFN-Sezione di Roma and University of Rome, "La Sapienza", I-00185 Rome, Italy

${ }^{\text {ai }}$ Nuclear Physics Institute, St. Petersburg, Russia

aj University and INFN, Salerno, I-84100 Salerno, Italy

ak University of California, San Diego, CA 92093, USA

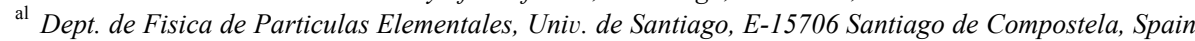

am Bulgarian Academy of Sciences, Central Lab. of Mechatronics and Instrumentation, BU-1113 Sofia, Bulgaria 
an Center for High Energy Physics, Adv. Inst. of Sciences and Technology, 305-701 Taejon, South Korea

${ }^{\text {ao }}$ University of Alabama, Tuscaloosa, AL 35486, USA

ap Utrecht University and NIKHEF, NL-3584 CB Utrecht, The Netherlands

aq Purdue University, West Lafayette, IN 47907, USA

${ }^{\text {ar }}$ Paul Scherrer Institut, PSI, CH-5232 Villigen, Switzerland

as DESY-Institut für Hochenergiephysik, D-15738 Zeuthen, Germany

${ }^{a}$ Eidgenössische Technische Hochschule, ETH Zürich, CH-8093 Zürich, Switzerland

au University of Hamburg, D-22761 Hamburg, Germany

${ }^{a v}$ National Central University, Chung-Li, Taiwan, ROC

${ }^{\text {aw }}$ Department of Physics, National Tsing Hua University, Taiwan, ROC

Received 21 May 1999

Editor: K. Winter

\begin{abstract}
The two-photon width $\Gamma_{\gamma \gamma}$ of the $\eta_{c}$ meson has been measured with the L3 detector at LEP. The $\eta_{c}$ is studied in the decay modes $\pi^{+} \pi^{-} \pi^{+} \pi^{-}, \pi^{+} \pi^{-} \mathrm{K}^{+} \mathrm{K}^{-}, \mathrm{K}_{s}^{0} \mathrm{~K}^{ \pm} \pi^{\mp}, \mathrm{K}^{+} \mathrm{K}^{-} \pi^{0}, \pi^{+} \pi^{-} \eta, \pi^{+} \pi^{-} \eta^{\prime}$, and $\rho^{+} \rho^{-}$using an integrated luminosity of $140 \mathrm{pb}^{-1}$ at $\sqrt{s} \simeq 91 \mathrm{GeV}$ and of $52 \mathrm{pb}^{-1}$ at $\sqrt{s} \simeq 183 \mathrm{GeV}$. The result is $\Gamma_{\gamma \gamma}\left(\eta_{c}\right)=6.9 \pm 1.7 \mathrm{stat} \pm 0.8 \mathrm{sys} \pm$ 2.0 (BR) keV. The $Q^{2}$ dependence of the $\eta_{c}$ cross section is studied for $Q^{2}<9 \mathrm{GeV}^{2}$. It is found to be better described by a Vector Meson Dominance model form factor with a J-pole than with a $\rho$-pole. In addition, a signal of $29 \pm 11$ events is observed at the $\chi_{\mathrm{c} 0}$ mass. Upper limits for the two-photon widths of the $\chi_{\mathrm{c} 0}, \chi_{\mathrm{c} 2}$, and $\eta_{c}^{\prime}$ are also given. (C) 1999 Elsevier Science B.V. All rights reserved.
\end{abstract}

\section{Introduction}

The study of resonance formation via two-photon interactions in $\mathrm{e}^{+} \mathrm{e}^{-}$colliders provides valuable information on the quark substructure of the meson. The cross section for two-photon resonance formation $\mathrm{e}^{+} \mathrm{e}^{-} \rightarrow \mathrm{e}^{+} \mathrm{e}^{-} \gamma^{*} \gamma^{*} \rightarrow \mathrm{e}^{+} \mathrm{e}^{-} R$, where $R$ is a $\mathrm{C}=+1$ meson, is given by [1]

$$
\sigma\left(\mathrm{e}^{+} \mathrm{e}^{-} \rightarrow \mathrm{e}^{+} \mathrm{e}^{-} R\right)=\int \sigma_{\gamma \gamma \rightarrow R} d L_{\gamma \gamma}\left(W^{2}\right)
$$

with the Breit-Wigner cross section

$$
\begin{aligned}
\sigma_{\gamma \gamma \rightarrow R}\left(W^{2}, q_{1}^{2}, q_{2}^{2}\right) \\
=8 \pi\left(2 J_{R}+1\right) \Gamma_{\gamma \gamma}(R) F^{2}\left(q_{1}^{2}, q_{2}^{2}\right) \\
\quad \times \frac{\Gamma_{R}}{\left(W^{2}-M_{R}^{2}\right)^{2}+M_{R}^{2} \Gamma_{R}^{2}} .
\end{aligned}
$$

Here, $W$ is the two-photon centre of mass energy, $q_{1}^{2}$ and $q_{2}^{2}$ are the squares of the virtual photon fourmomenta, and $L_{\gamma \gamma}\left(W^{2}\right)$ is the two-photon luminos-

\footnotetext{
${ }^{1}$ Also supported by CONICET and Universidad Nacional de La Plata, CC 67, 1900 La Plata, Argentina.

2 Also supported by Panjab University, Chandigarh-160014, India.

${ }^{3}$ Supported by Deutscher Akademischer Austauschdienst.

${ }^{4}$ Also supported by the Hungarian OTKA fund under contract numbers T22238 and T026178.

${ }^{5}$ Supported by the German Bundesministerium für Bildung, Wissenschaft, Forschung und Technologie.

${ }^{6}$ Supported by the National Natural Science Foundation of China.

${ }^{7}$ Supported by the Hungarian OTKA fund under contract numbers T019181, F023259 and T024011.

${ }^{8}$ Supported also by the Comisión Interministerial de Ciencia y Tecnología.
} 
ity function. The resonance $R$ is characterised by the mass $M_{R}$, total spin $J_{R}$, total width $\Gamma_{R}$ and the two-photon partial width $\Gamma_{\gamma \gamma}(R)$. The two-photon width and the transition form factor $F^{2}\left(q_{1}^{2}, q_{2}^{2}\right)$ are the two parameters to be measured.

In two-photon collisions, the scattered electron and positron emerge with a small energy loss and an almost unmodified direction after having radiated one photon each. They therefore usually go undetected along the beam direction, allowing the photons to be considered as 'quasi-real', with $q^{2} \simeq 0$. At $q_{1}^{2} \simeq q_{2}^{2} \simeq 0$, the form factor $F^{2}\left(q_{1}^{2}, q_{2}^{2}\right)$ is normalised to unity, leaving the two-photon width as the only unknown parameter, linearly proportional to the total cross section:

$\sigma\left(\mathrm{e}^{+} \mathrm{e}^{-} \rightarrow \mathrm{e}^{+} \mathrm{e}^{-} R\right)=\kappa \Gamma_{\gamma \gamma}(R)$.

If the scattered electron or positron is detected at small angles, the event is said to be tagged: one photon has $Q^{2}=-q^{2}$ while the other is quasi-real with $q^{2} \simeq 0$. Using tagged events, the transition form factor can be measured. It is parametrised in the Vector Meson Dominance (VMD) model by a pole form

$F\left(Q^{2}\right)=\frac{1}{1+Q^{2} / \Lambda^{2}}$,

with $\Lambda^{2}=M_{V}^{2}, \quad V=\rho, \omega, \phi, \mathrm{J} \ldots$

In the case of charmonium $(c \bar{c})$ mesons, the $J^{P C}$ states accessible in quasi-real two-photon reactions are $0^{-+}, 0^{++}$and $2^{++}$, corresponding to $\eta_{c}(2979)$, $\chi_{\mathrm{c} 0}(3417)$ and $\chi_{\mathrm{c} 2}(3556)$. The formation of the $\chi_{\mathrm{c} 1}(3510), \mathrm{a}^{++}$state, is forbidden for two real photons according to the Landau-Yang theorem [2]. The radial recurrence $\eta_{c}^{\prime}(3594)$ has been observed by the Crystal Ball experiment [3] in the process $\psi^{\prime} \rightarrow$ $\gamma \eta_{c}^{\prime}$, but not in two-photon collisions [4].

In this paper we report on the study of the formation of the $\eta_{c}$. The two-photon width and the $Q^{2}$ dependence of the cross section are determined. Theoretical calculations, based on the assumption that the two heavy charm quarks are bound together by a QCD potential, predict 3-9 keV for the $\eta_{c}$ two-photon width [5]. The $Q^{2}$ dependence of the cross section has also been calculated [6,7]. It contains information about the quark momentum distribution inside the meson, and its shape is predicted to be well described by a VMD model form factor with $M_{V}=M_{\mathrm{J}}$. The $Q^{2}$ dependence of the $\eta_{c}$ cross section has not been measured up to now.

We report results from the data obtained with the L3 detector at centre of mass energies $\sqrt{s} \simeq 91 \mathrm{GeV}$, with a total integrated luminosity of $140.2 \mathrm{pb}^{-1}$, and at $\sqrt{s} \simeq 183 \mathrm{GeV}$, with an integrated luminosity of $52.4 \mathrm{pb}^{-1}$. This data sample includes the $30 \mathrm{pb}^{-1}$ which we used for our previous measurement of $\Gamma_{\gamma \gamma}\left(\eta_{c}\right)$ [8]. Since the $\eta_{c}$ resonance does not have a dominant decay mode, nine different decay modes with branching ratios ranging from $0.5 \%$ to $2 \%$ have been analysed. They are listed in Table 1 together with the branching ratio of each channel, derived from Ref. [9].

Table 1

The branching ratio BR (derived from Ref. [9]), the efficiencies $\epsilon_{\sqrt{s}}$, and the two-photon widths as obtained with an unbinned likelihood fit, at $\sqrt{s} \simeq 91 \mathrm{GeV}$ and $\sqrt{s} \simeq 183 \mathrm{GeV}$ for the different $\eta_{c}$ decay modes considered in the analysis. All branching ratios contain a common error of $28 \%$ due to the uncertainty in $\operatorname{BR}\left(\mathrm{J} \rightarrow \gamma \eta_{c}\right)$

\begin{tabular}{|c|c|c|c|c|c|}
\hline Decay mode & $\mathrm{BR}(\%)$ & $\epsilon_{91}(\%)$ & $\epsilon_{183}(\%)$ & $\Gamma_{\gamma \gamma}^{91}(\mathrm{keV})$ & $\Gamma_{\gamma \gamma}^{183}(\mathrm{keV})$ \\
\hline$\eta_{c} \rightarrow \pi^{+} \pi^{-} \pi^{+} \pi^{-}$ & $1.2 \pm 0.4$ & 4.9 & 4.1 & $<28$ & $<36$ \\
\hline$\eta_{c} \rightarrow \mathrm{K}^{+} \mathrm{K}^{-} \pi^{+} \pi^{-}$ & $2.0 \pm 0.7$ & 5.2 & 4.5 & $10 \pm 8$ & $16 \pm 10$ \\
\hline$\eta_{c} \rightarrow \mathrm{K}_{s}^{0} \mathrm{~K}^{ \pm} \pi^{\mp}$ & $1.3 \pm 0.4$ & 5.1 & 5.0 & $5.4_{-3.4}^{+3.9}$ & $5.5_{-3.9}^{+4.7}$ \\
\hline$\eta_{c} \rightarrow \pi^{+} \pi^{-} \eta(\gamma \gamma)$ & $1.3 \pm 0.5$ & 3.3 & 3.9 & $7.4_{-4.4}^{+4.4}$ & $<12$ \\
\hline$\eta_{c} \rightarrow \mathrm{K}^{+} \mathrm{K}^{-} \pi^{0}$ & $0.9 \pm 0.3$ & 2.6 & 3.3 & $21_{-11}^{+12.4}$ & $<34$ \\
\hline$\eta_{c} \rightarrow \rho^{+} \rho^{-}$ & $1.7 \pm 0.6$ & 0.8 & 0.9 & $<28$ & $24_{-17}^{+21}$ \\
\hline$\eta_{c} \rightarrow \pi^{+} \pi^{-} \eta\left(\pi^{+} \pi^{-} \pi^{0}\right)$ & $0.8 \pm 0.3$ & 1.6 & 1.2 & $<16$ & $15_{-11}^{+18}$ \\
\hline$\eta_{c} \rightarrow \pi^{+} \pi^{-} \eta^{\prime}\left(\pi^{+} \pi^{-} \eta\right)$ & $0.5 \pm 0.2$ & 2.8 & 2.5 & $7_{-6}^{+7}$ & $<32$ \\
\hline$\eta_{c} \rightarrow \pi^{+} \pi^{-} \eta^{\prime}(\rho \gamma)$ & $0.8 \pm 0.3$ & 3.0 & 2.5 & $16 \pm 10$ & $<29$ \\
\hline
\end{tabular}




\section{L3 detector and Monte Carlo}

A detailed description of the L3 detector can be found in Ref. [10]. The analysis described in this paper is mainly based on the central tracking system and the high resolution electromagnetic calorimeter.

Particles scattered at small angles are measured by the luminosity monitors (LUMI), covering a polar angle range $26 \mathrm{mrad}<\theta<65 \mathrm{mrad}$ on each side of the detector. For data at $\sqrt{s} \simeq 183 \mathrm{GeV}$, particles can also be detected by the very small angle tagger (VSAT) covering a polar angle range between 5 $\mathrm{mrad}<\theta<8 \mathrm{mrad}$ for an azimuthal angle range of $-0.8 \mathrm{rad}<\phi<0.8 \mathrm{rad}$ or $\pi-0.8 \mathrm{rad}<\phi<\pi+$ $0.8 \mathrm{rad}[11]$.

The two-photon events are collected predominantly by a track trigger [12] which requires at least two charged particles with transverse momentum $p_{\mathrm{t}}>150 \mathrm{MeV}$, back to back, in the plane transverse to the beam, within $\pm 41^{\circ}$ for data at $\sqrt{s} \simeq 91 \mathrm{GeV}$, and within $\pm 60^{\circ}$ for data at $\sqrt{s} \simeq 183 \mathrm{GeV}$. In addition, there is a trigger for electron tags, which requires an energy deposit of at least $70 \%$ of the beam energy in the LUMI, in coincidence with at least one charged track in the central tracker.

In order to compute the acceptance and efficiency of the detector we have used the PC Monte Carlo [13], based on the formalism of Budnev et al. [1]. The $Q^{2}$ dependence of the cross section is taken into account using a VMD model form factor.

The particles produced in the reaction are followed through the different L3 subdetectors with the GEANT [14] simulation program and the events are reconstructed and analysed in the same way as real data.

\section{Selection criteria}

The $\eta_{c}$ decays, listed in Table 1 , result in final states with either two or four charged tracks with charge balance, accompanied by zero, one, two or four photons.

A track must have at least 18 out of a maximum of 62 hits in the central tracker. The distance of closest approach to the beam line in the transverse plane is required to be less than $3 \mathrm{~mm}$, except for tracks associated with a $\mathrm{K}_{s}^{0}$ decay. In order to remove electrons, we require for tracks with a momentum $p$ larger than $0.8 \mathrm{GeV}$ that $E / p<0.9$, where $E$ is the associated energy in the electromagnetic calorimeter.

A photon candidate is an electromagnetic cluster separated from all tracks by at least $100 \mathrm{mrad}$ in $\phi$ and $140 \mathrm{mrad}$ in $\theta$. In the search for $\pi^{0}$ candidates, the two photons must both have an energy of at least $50 \mathrm{MeV}$. To reduce the combinatorial background under the $\pi^{0}$ signal, the angle $\psi_{\gamma \gamma}$ between the photons of the $\pi^{0}$ candidate must satisfy $\cos \psi_{\gamma \gamma}>$ 0.6. The effective mass of the two photons must be within $20 \mathrm{MeV}$ of the $\pi^{0}$ mass if both photons are in the barrel part of the electromagnetic calorimeter $\left(42^{\circ}<\theta<138^{\circ}\right)$, and within $30 \mathrm{MeV}$ otherwise. The $\pi^{0}$ signal is shown in Fig. 1a and 1b. The two photons of an $\eta$ candidate must have an energy of at least $100 \mathrm{MeV}$, and at least one of them must be in the barrel. In addition, it is required that $\cos \psi_{\gamma \gamma}>$

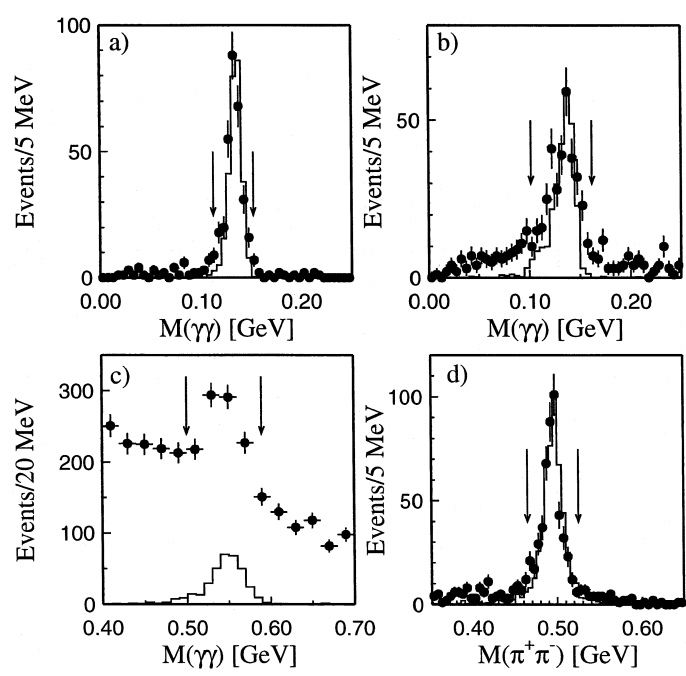

Fig. 1. (a) The invariant mass of two photons in the $\mathrm{K}^{+} \mathrm{K}^{-} \pi^{0}$ sample, with both photons in the barrel $\left(42^{\circ}<\theta<138^{\circ}\right)$ after all other cuts have been performed. The events between the arrows are accepted. (b) The same, with at least one of the photons in the endcaps $\left(12^{\circ}<\theta<38^{\circ}\right.$ and $\left.142^{\circ}<\theta<168^{\circ}\right)$. (c) The invariant mass of two photons in the $\pi^{+} \pi^{-} \eta$ sample, after all other cuts have been performed. (d) The invariant mass of the two tracks associated with a secondary vertex in the $\mathrm{K}_{s}^{0} \mathrm{~K}^{ \pm} \pi^{\mp}$ sample, selected with the cuts described in the text, after all other cuts have been made. In all plots the points with error bars represent the data, while the histogram represents the $\eta_{c}$ Monte Carlo. The normalisation of the Monte Carlo is arbitrary. 
-0.7 , and that the $\gamma \gamma$ effective mass is within 45 $\mathrm{MeV}$ of the $\eta$ mass, see Fig. $1 \mathrm{c}$.

If a cluster with at least $70 \%$ of the beam energy is found in the LUMI calorimeter or a cluster with more than $50 \%$ of the beam energy is found in the VSAT, the event is classified as tagged.

To ensure that no final state particle of the resonance decay has escaped detection, the squared vectorial sum of the transverse momenta of all detected particles, $\left(\Sigma \boldsymbol{p}_{\mathrm{t}}\right)^{2}$, is required to be smaller than 0.1 $\mathrm{GeV}^{2}$. If a tag is found, it is also included in this sum. However, if the $\left(\Sigma \boldsymbol{p}_{\mathrm{t}}\right)^{2}$ excluding the tag is smaller than the $\left(\Sigma \boldsymbol{p}_{\mathrm{t}}\right)^{2}$ with the tag included, the event is considered as untagged. Additionally, for events with one or more photons in the final state, the $\left(\Sigma \boldsymbol{p}_{\mathrm{t}}\right)^{2}$ excluding the photon(s) should be larger than $0.005 \mathrm{GeV}^{2}$.

Additional cuts for each decay mode are listed below. The masses of the intermediate states which are used in the cuts are taken from the observed central values of the peaks.

- $\eta_{c} \rightarrow \pi^{+} \pi^{-} \pi^{+} \pi^{-}$or $\pi^{+} \pi^{-} \mathrm{K}^{+} \mathrm{K}^{-}$

These events leave four tracks and no photons in the detector. Using the $d E / d x$ measurement in the central tracking system, a $\chi^{2}$ to be a pion or kaon can be calculated for each track, and thus a combined probability that the four tracks are $\pi^{+} \pi^{-} \pi^{+} \pi^{-}, \mathrm{K}^{+} \mathrm{K}^{-} \pi^{+} \pi^{-}$, or $\mathrm{K}^{+} \mathrm{K}^{-} \mathrm{K}^{+} \mathrm{K}^{-}$ can be derived. For a $\pi^{+} \pi^{-} \pi^{+} \pi^{-}$event, the $\pi^{+} \pi^{-} \pi^{+} \pi^{-}$probability divided by the sum of $\pi^{+} \pi^{-} \pi^{+} \pi^{-}, \mathrm{K}^{+} \mathrm{K}^{-} \pi^{+} \pi^{-}$, and $\mathrm{K}^{+} \mathrm{K}^{-} \mathrm{K}^{+} \mathrm{K}^{-}$ probabilities must be larger than $55 \%$. For a $\mathrm{K}^{+} \mathrm{K}^{-} \pi^{+} \pi^{-}$event, the $\mathrm{K}^{+} \mathrm{K}^{-} \pi^{+} \pi^{-}$probability divided by the sum of $\pi^{+} \pi^{-} \pi^{+} \pi^{-}$, $\mathrm{K}^{+} \mathrm{K}^{-} \pi^{+} \pi^{-}$, and $\mathrm{K}^{+} \mathrm{K}^{-} \mathrm{K}^{+} \mathrm{K}^{-}$probabilities must be larger than $55 \%$. Events that do not fall in either category are rejected. The $d E / d x$ measurement has a good $\pi / K$ separating power for tracks with a momentum $p<0.5 \mathrm{GeV}$. As the momenta of the decay particles of the $\eta_{c}$ extend to $2 \mathrm{GeV}$, there exists some misidentification, which is estimated using Monte Carlo. Out of all $\eta_{c} \rightarrow \pi^{+} \pi^{-} \pi^{+} \pi^{-}$Monte Carlo events that are selected, $21 \%$ are identified wrongly as $\mathrm{K}^{+} \mathrm{K}^{-} \pi^{+} \pi^{-}$, and of all $\eta_{c} \rightarrow \mathrm{K}^{+} \mathrm{K}^{-} \pi^{+} \pi^{-}$ Monte Carlo events that are selected, $8 \%$ are wrongly identified as $\pi^{+} \pi^{-} \pi^{+} \pi^{-}$.

- $\eta_{c} \rightarrow \mathrm{K}_{s}^{0} \mathrm{~K}^{ \pm} \pi^{\mp}$
For this final state the geometrical reconstruction of the secondary vertex $\mathrm{K}_{s}^{0} \rightarrow \pi^{+} \pi^{-}$requires two oppositely charged tracks, each with a radial distance from the interaction point in the transverse plane greater than $1 \mathrm{~mm}$. The two tracks must form a secondary vertex more than $3 \mathrm{~mm}$ away from the interaction point. The angle between the tracks must be smaller than $2.5 \mathrm{rad}$. If the angle between the $\mathrm{K}_{s}^{0}$ line of flight and the sum of the two track momenta is greater than 60 $\mathrm{mrad}$, the $\mathrm{K}_{s}^{0}$ candidate is rejected. Finally, the invariant mass of the two pions must be within 30 $\mathrm{MeV}$ of the $\mathrm{K}_{s}^{0}$ mass, see Fig. 1d.

- $\eta_{c} \rightarrow \pi^{+} \pi^{-} \eta, \eta \rightarrow \gamma \gamma$

For this decay mode two photons, reconstructing an $\eta$, and two tracks must be observed in the detector.

- $\eta_{c} \rightarrow \mathrm{K}^{+} \mathrm{K}^{-} \pi^{0}$

For this decay mode two photons, reconstructing a $\pi^{0}$, and two tracks must be observed in the detector. Since there exists no background from $\eta_{c} \rightarrow \pi^{+} \pi^{-} \pi^{0}$, only a loose cut on the $d E / d x$ is used in order to keep the efficiency high: the $\mathrm{K}^{+} \mathrm{K}^{-}$probability divided by the sum of the $\mathrm{K}^{+} \mathrm{K}^{-}$and $\pi^{+} \pi^{-}$probabilities must be larger than $30 \%$.

- $\eta_{c} \rightarrow \rho^{+} \rho^{-}, \rho^{ \pm} \rightarrow \pi^{ \pm} \pi^{0}$

Four photons are combined in order to find two $\pi^{0}$ candidates, which are combined with the two tracks to form two charged $\rho$ mesons. The event is accepted if both combinations satisfy $\left|M\left(\pi^{ \pm} \pi^{0}\right)-M\left(\rho^{ \pm}\right)\right|<0.25 \mathrm{GeV}$.

- $\eta_{c} \rightarrow \pi^{+} \pi^{-} \eta, \eta \rightarrow \pi^{+} \pi^{-} \pi^{0}$

For this decay mode the cut on the angle between the two photons is widened to $\cos \psi_{\gamma \gamma}>0.4$. Two out of the four observed tracks must combine with the $\pi^{0}$ candidate to form an $\eta$, with $\left|M\left(\pi^{+} \pi^{-} \pi^{0}\right)-M(\eta)\right|<40 \mathrm{MeV}$.

- $\eta_{c} \rightarrow \pi^{+} \pi^{-} \eta^{\prime}, \eta^{\prime} \rightarrow \pi^{+} \pi^{-} \eta, \eta \rightarrow \gamma \gamma$

For this decay mode two photons, reconstructing an $\eta$, and four tracks must be observed in the detector. The $\eta$ candidate must combine with two of the tracks to form an $\eta^{\prime}$, with $\mid M\left(\pi^{+} \pi^{-} \eta\right)-$ $M\left(\eta^{\prime}\right) \mid<50 \mathrm{MeV}$.

- $\eta_{c} \rightarrow \pi^{+} \pi^{-} \eta^{\prime}, \eta^{\prime} \rightarrow \rho^{0} \gamma, \rho^{0} \rightarrow \pi^{+} \pi^{-}$

To select this decay mode, $\rho^{0}$ candidates, formed by a pair of tracks with $0.60 \mathrm{GeV}<M\left(\pi^{+} \pi^{-}\right)<$ $0.92 \mathrm{GeV}$, are combined with a photon, with 
energy greater than $100 \mathrm{MeV}$, to form an $\eta^{\prime}$, with $\left|M\left(\rho^{0} \gamma\right)-M\left(\eta^{\prime}\right)\right|<60 \mathrm{MeV}$ and $\cos \psi_{\rho^{0} \gamma}>$ -0.3 .

The selection efficiencies for the analysed decay modes are given in Table 1 , for both data samples at $\sqrt{s} \simeq 91 \mathrm{GeV}$ and at $\sqrt{s} \simeq 183 \mathrm{GeV}$.

The mass distribution of the selected untagged events is presented in Fig. 2. A fit of this spectrum in the mass range $2.4 \mathrm{GeV}<M<3.2 \mathrm{GeV}$, with an exponential background plus a Gaussian for the signal, gives for the peak position $M\left(\eta_{c}\right)=2.974 \pm$ $0.018 \mathrm{GeV}$, in excellent agreement with the world average $2.979 \pm 0.002 \mathrm{GeV}$ [9]. The width of the Gaussian is $57 \pm 16 \mathrm{MeV}$, consistent with the reconstructed width in the Monte Carlo of $70 \mathrm{MeV}$. The area of the peak corresponds to a total of $93 \pm 33 \eta_{c}$ events.

The $\chi_{\mathrm{c} 0}(3417)$ and $\chi_{\mathrm{c} 2}(3556)$ are also known to decay into $\pi^{+} \pi^{-} \pi^{+} \pi^{-}$and $\mathrm{K}^{+} \mathrm{K}^{-} \pi^{+} \pi^{-}$. Their branching ratios to the other analysed decay modes are unknown. Therefore the mass spectrum has been divided into two parts in Fig. 3: Fig. 3a shows the mass distribution of the events with one or more photons or $\mathrm{a} \mathrm{K}_{s}^{0}$ in the final state, while Fig. $3 \mathrm{~b}$ shows the mass spectrum of the $\pi^{+} \pi^{-} \pi^{+} \pi^{-}$and $\mathrm{K}^{+} \mathrm{K}^{-} \pi^{+} \pi^{-}$events. However, in Fig. 3b, no enhancements are visible around the $\chi_{\mathrm{c} 0}$ and $\chi_{\mathrm{c} 2}$ masses. In Fig. $3 a$, on the other hand, an enhance-

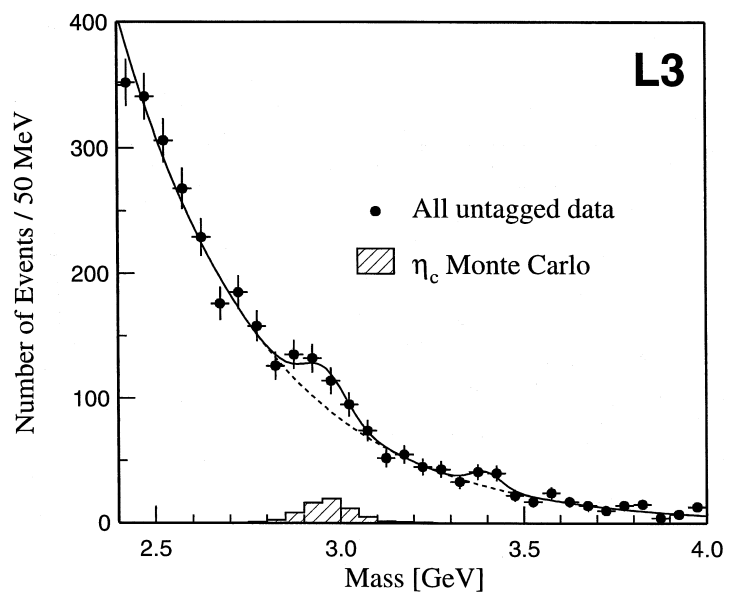

Fig. 2. The mass distribution of all selected untagged events. The hatched histogram is the arbitrarily scaled $\eta_{c}$ Monte Carlo. The solid lines represent a fit to the data with an exponential background and Gaussians for the $\eta_{c}$ and $\chi_{\mathrm{c} 0}$ signals.

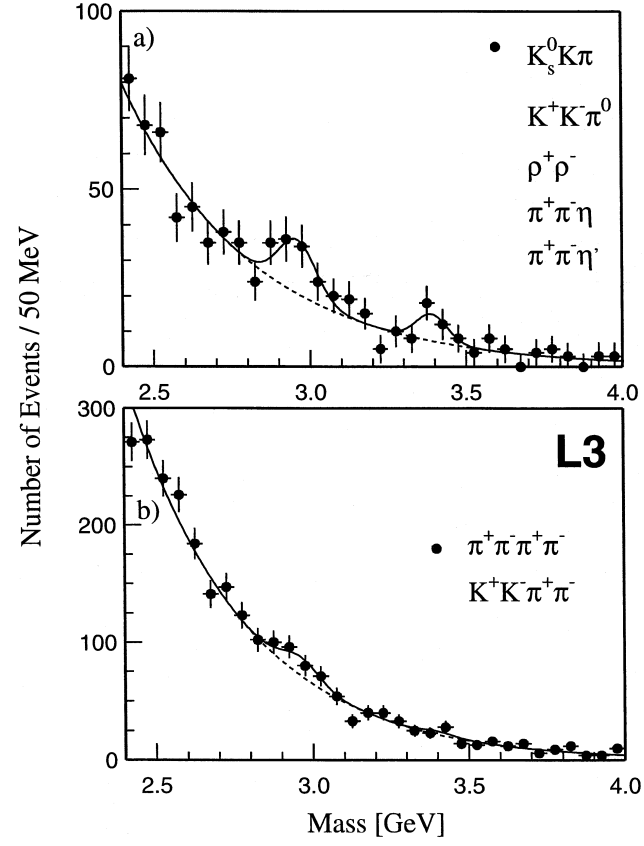

Fig. 3. The mass distribution of the selected untagged events (a) for the decay modes $\mathrm{K}_{s}^{0} \mathrm{~K}^{ \pm} \pi^{\mp}, \mathrm{K}^{+} \mathrm{K}^{-} \pi^{0}, \rho^{+} \rho^{-}, \pi^{+} \pi^{-} \eta$, and $\pi^{+} \pi^{-} \eta^{\prime}$, (b) for the decay modes $\pi^{+} \pi^{-} \pi^{+} \pi^{-}$and $\mathrm{K}^{+} \mathrm{K}^{-} \pi^{+} \pi^{-}$. The solid lines represent fits to the data with an exponential background and Gaussians for the $\eta_{c}$ and $\chi_{\mathrm{c} 0}$ signals.

ment is visible around $3.4 \mathrm{GeV}$. A fit to this enhancement in the mass range $3.2 \mathrm{GeV}<M<4 \mathrm{GeV}$ gives for the peak position $3.400 \pm 0.019 \mathrm{GeV}$, in agreement with the world average $\chi_{\mathrm{c} 0}$ mass $M\left(\chi_{\mathrm{c} 0}\right)$ $=3.417 \pm 0.003 \mathrm{GeV}$, and for the width $58 \pm 18$ $\mathrm{MeV}$, in agreement with the reconstructed width from the Monte Carlo of $70 \mathrm{MeV}$. The area of the fitted Gaussian is $29 \pm 11$ events. A similar fit to Fig. $3 \mathrm{~b}$ yields only $7 \pm 6$ events above background.

\section{The two-photon width}

Since the $\eta_{c}$ signal has low statistics in each decay mode, the two-photon width is obtained with a combined unbinned likelihood fit. The fit takes into account the different weight of each decay mode due to the different background levels, efficiencies, branching ratios, and the different integrated luminosities at $\sqrt{s} \simeq 91 \mathrm{GeV}$ and at $\sqrt{s} \simeq 183 \mathrm{GeV}$. All mass spectra are fitted simultaneously with a sum of 
a normalised exponential background function $b_{i}(x)$ and a Gaussian distribution $g_{i}(x)$, where $i$ runs over the different decay modes and the two centre-of-mass energies considered:

$f_{i}(x)=\left(1-p_{i}\right) b_{i}(x)+p_{i} g_{i}(x)$.

Here, $p_{i}$ is the ratio of the number of signal events, $S_{i}$, over the total number of events in mass spectrum $i$. The exponential background, $b_{i}(x)$, is allowed to vary for each spectrum, but the number of signal events in the Gaussian for each spectrum is related to the two-photon width by

$S_{i}=\epsilon_{i} \mathscr{L} \mathrm{BR}_{i} \kappa \Gamma_{\gamma \gamma}$.

Here, $\epsilon_{i}$ is the total efficiency, $\mathscr{L}$ is the integrated luminosity, and $\mathrm{BR}_{i}$ is the branching ratio for decay mode $i$. The proportionality factor $\kappa$ is obtained from Monte Carlo, using Eq. (3). The events in $\chi_{\mathrm{c} 0}$ signal region (between 3.3 and $3.5 \mathrm{GeV}$ ) are excluded from the fit. For the $\mathrm{K}^{+} \mathrm{K}^{-} \pi^{+} \pi^{-}$and $\pi^{+} \pi^{-} \pi^{+} \pi^{-}$mass spectra, the whole region $M>$ $3.2 \mathrm{GeV}$ is excluded, where the $\chi_{\mathrm{c} 0}$ signal, the $\chi_{\mathrm{c} 2}$ signal, and small enhancements due to $\pi / K$ misidentification are expected. The effect of this cut is taken into account in the systematic error. The $\eta_{c}$ two-photon width obtained from the fit is $6.9 \pm$ 1.7 stat $\mathrm{keV}$. It corresponds to a total of $76 \pm 19 \eta_{c}$ events, in agreement with the number of events obtained with the fit to the total mass spectrum shown in Fig. 2. The two-photon widths or upper limits for the individual decay modes are also given in Table 1. They have been obtained with an unbinned likelihood fit to the individual spectra using an exponential background and a Gaussian for the signal, with the Gaussian position and width fixed to the Monte Carlo values.

The systematic error related to the selection requirements includes contributions from the cut on the number of hits on a track $(5 \%)$, from the $d E / d x$ cuts (3\%), and from the $\pi^{0}$ selection (4\%). Furthermore, the systematic errors take into account uncertainties on the trigger efficiency $(2 \%)$, on the Monte Carlo statistics $(2 \%)$, on the background subtraction $(3 \%)$ and on the uncertainty on the individual branching ratios $(9 \%)$. They add up to $12 \%$, resulting in a systematic error on $\Gamma_{\gamma \gamma}\left(\eta_{c}\right)$ of $0.8 \mathrm{keV}$. The uncertainty introduced by the poor knowledge of the branching ratio $\mathrm{BR}\left(\mathrm{J} \rightarrow \eta_{c} \gamma\right)=(1.27 \pm 0.36) \%$
Table 2

Summary of the published measurements of $\Gamma_{\gamma \gamma}\left(\eta_{c}\right)[16,8]$

\begin{tabular}{ll}
\hline Experiment & $\Gamma_{\gamma \gamma}\left(\eta_{c}\right)(\mathrm{keV})$ \\
\hline PLUTO & $28 \pm 15$ \\
TPC $/ 2 \gamma$ & $6.4_{-3.4}^{+5.0}$ \\
CLEO & $5.9_{-1.8}^{+2.1} \pm 1.9$ \\
L3 & $8.0 \pm 2.4 \pm 2.3$ \\
ARGUS & $11.3 \pm 4.2$ \\
E760 & $6.7_{-1.7}^{+2.4} \pm 2.3$ \\
L3 (this analysis) & $6.9 \pm 1.7 \pm 0.8 \pm 2.0$ \\
\hline
\end{tabular}

[15], which is contained in all branching ratio uncertainties, leads to an additional error on $\Gamma_{\gamma \gamma}\left(\eta_{c}\right)$ of 2.0 $\mathrm{keV}$.

In Table 2 our measurement is compared to previous measurements $[16,8]$ of the $\eta_{c}$ two-photon width. It is found to be in good agreement. The measurement is also in good agreement with the theoretical predictions [5].

If we assume that the excess of events at $3.4 \mathrm{GeV}$ is due to $\chi_{\mathrm{c} 0}$ formation, the $\chi_{\mathrm{c} 0}$ two-photon width can be obtained using a similar fit as for the $\eta_{c}$. However, only the $\pi^{+} \pi^{-} \pi^{+} \pi^{-}$and $\mathrm{K}^{+} \mathrm{K}^{-} \pi^{+} \pi^{-}$ events, for which the $\chi_{\mathrm{c} 0}$ branching ratios are known, can be included in the fit. Since the number of signal events is low for these decay modes, it is only possible to set a $95 \%$ C.L. upper limit $\Gamma_{\gamma \gamma}\left(\chi_{\mathrm{c} 0}\right)<5.5$ $\mathrm{keV}$. This is consistent with the upper limit by CLEO, $\Gamma_{\gamma \gamma}\left(\chi_{\mathrm{c} 0}\right)<6.2 \mathrm{keV}$, and the measurement by the Crystal Ball experiment, $\Gamma_{\gamma \gamma}\left(\chi_{\mathrm{c} 0}\right)=4.0 \pm 2.8$ $\mathrm{keV}$ [17].

Also the $\chi_{\mathrm{c} 2}(3556)$ has known branching ratios into $\pi^{+} \pi^{-} \pi^{+} \pi^{-}$and $\mathrm{K}^{+} \mathrm{K}^{-} \pi^{+} \pi^{-}$. No signal is observed in Fig. $3 \mathrm{~b}$. We obtain for the $\chi_{\mathrm{c} 2}$ two-photon width a $95 \%$ C.L. upper limit of $1.4 \mathrm{keV}$, consistent with our previous measurement [18].

We also observe no signal for the $\eta_{c}^{\prime}(3594)$. Ref. [19] predicts the hadronic branching ratios of the $\eta_{c}$ and $\eta_{c}^{\prime}$ to be about equal. If we assume that the efficiencies and branching ratios of the $\eta_{c}^{\prime}$ are the same as for the $\eta_{c}$ for the analysed final states, we obtain $\Gamma_{\gamma \gamma}\left(\eta_{c}^{\prime}\right)<2.0 \mathrm{keV}$ at $95 \%$ C.L.

\section{The $\eta_{c}$ form factor}

The $\eta_{c}$ transition form factor, as defined in Eq. (2), can be studied using tagged events. The mass 


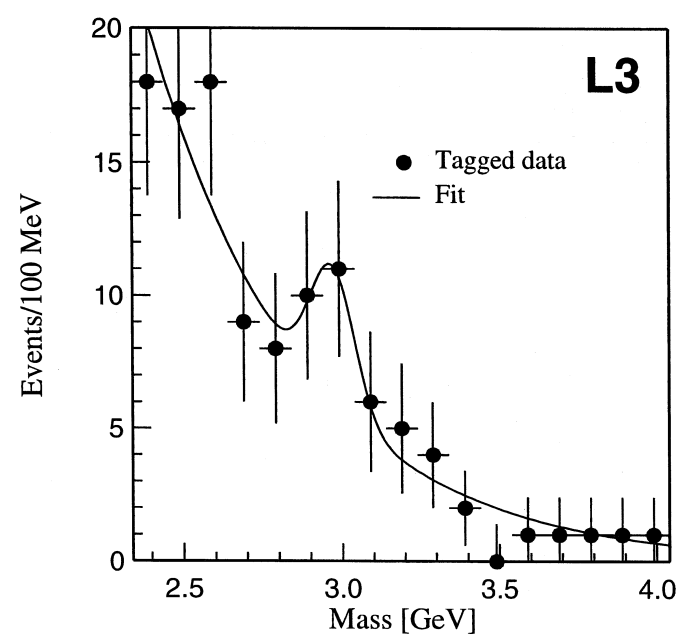

Fig. 4. The mass distribution for selected events with a tag in the LUMI at $\sqrt{s} \simeq 91 \mathrm{GeV}$, or with a tag in the VSAT at $\sqrt{s} \simeq 183$ $\mathrm{GeV}$. The line is a fit with an exponential background and a Gaussian for the signal, with the mass and the width of the Gaussian fixed to the $\eta_{c}$ Monte Carlo prediction.

spectrum of the events with an electron found in the LUMI at $\sqrt{s} \simeq 91 \mathrm{GeV}$ or in the VSAT at $\sqrt{s} \simeq 183$ $\mathrm{GeV}$ is shown in Fig. 4. The number of events with a tag in the LUMI at $\sqrt{s} \simeq 183 \mathrm{GeV}$ is too low to contribute to the measurement. At $\sqrt{s} \simeq 91 \mathrm{GeV}$, the LUMI covers the $Q^{2}$ range from $1.3 \mathrm{GeV}^{2}$ to 8.5 $\mathrm{GeV}^{2}$. The VSAT covers at $\sqrt{s} \simeq 183 \mathrm{GeV}$ the $Q^{2}$ range between $0.2 \mathrm{GeV}^{2}$ and $0.8 \mathrm{GeV}^{2}$. The solid line in Fig. 4 represents a fit with an exponential for the background and a Gaussian for the signal, with the position and width of the $\eta_{c}$ Gaussian fixed to the Monte Carlo values. The area of the Gaussian is $8.3_{-4.9}^{+5.5}$ events.

We assume that the shape of the $\eta_{c}$ form factor is described by the Vector Meson Dominance model form factor given in Eq. (4). We compare three hypotheses for the pole mass $\Lambda$ : a $\rho$-pole, which is found to be a good description of the $Q^{2}$ dependence of the $\pi^{0}, \eta$, and $\eta^{\prime}$ cross sections [20], a J-pole, which is predicted to be a good approximation of the $Q^{2}$ dependence of the $\eta_{c}$ cross section [6,7], and an infinite pole or flat form factor, i.e. the $\gamma \gamma \rightarrow \eta_{c}$ cross section has no $Q^{2}$ dependence. Since our $\eta_{c}$ Monte Carlo has been generated with a J-pole, the Monte Carlo events are reweighted to simulate a $\rho$-pole or flat form factor.
From Eqs. (1) and (2) it can be seen that the cross section is proportional to the product of the two-photon width and the form factor. In order to measure the form factor in a $Q^{2}$-interval $\Delta Q^{2}$, the ratio $\sigma_{\text {data }}\left(\Delta Q^{2}\right) / \Gamma_{\gamma \gamma}$ has to be determined. The two-photon width $\Gamma_{\gamma \gamma}$ has already been measured using untagged events, and the cross section $\sigma_{\text {data }}\left(\Delta Q^{2}\right)$ can be obtained using the tagged events. Note that in the ratio $\sigma_{\text {data }}\left(\Delta Q^{2}\right) / \Gamma_{\gamma \gamma}$ almost all systematic errors cancel, in particular the error due to the uncertainty in $\operatorname{BR}\left(\mathrm{J} \rightarrow \eta_{c} \gamma\right)$.

The two cross sections $\sigma_{\text {data }}\left(\Delta Q^{2}\right)$, for events with a tag in the LUMI and for events with a tag in the VSAT, are obtained using an unbinned likelihood fit. The fit is similar to the one used to obtain the two-photon width, with the number of tagged signal events in each spectrum, $S_{i}$, equal to $\epsilon_{i}\left(\Delta Q^{2}\right)$ $\mathscr{L} \mathrm{BR}_{i} \sigma_{\text {data }}\left(\Delta Q^{2}\right)$. The efficiencies per $Q^{2}$ interval, $\epsilon_{i}\left(\Delta Q^{2}\right)$, have an uncertainty due to the choice of the form factor in the Monte Carlo less than 3\%. The cross sections correspond to $7.7 \pm 3.0$ events with a tag in the LUMI and $2.3 \pm 2.3$ events with a tag in the VSAT.

The $\sigma_{\text {data }}\left(\Delta Q^{2}\right) / \Gamma_{\gamma \gamma}$ ratios are given in Table 3, together with the Monte Carlo predictions for the three form factor hypotheses. A $\chi^{2}$ representing how well the measured $\sigma_{\text {data }}\left(\Delta Q^{2}\right) / \Gamma_{\gamma \gamma}$ correspond to the Monte Carlo predictions is calculated and the corresponding probabilities are given in the last column of Table 3 for each form factor hypothesis. The changes in the $\chi^{2}$ probabilities due to the variation

Table 3

Results for the tagged $\eta_{c}$ cross section $\sigma_{\text {data }}\left(\Delta Q^{2}\right) / \Gamma_{\gamma \gamma}\left(\eta_{c}\right)$ for events with a tag in the LUMI at $\sqrt{s} \simeq 91 \mathrm{GeV}$ and with a tag in the VSAT at $\sqrt{s} \simeq 183 \mathrm{GeV}$, compared to the Monte Carlo predictions for a $\rho$-pole form factor, a J-pole form factor, and a flat form factor. In the last column the $\chi^{2}$ probability that the data and the Monte Carlo are compatible is given for each form factor hypothesis. Between parentheses, the $\chi^{2}$ probabilities if $\Gamma_{\gamma \gamma}$ is varied from $5.2 \mathrm{keV}$ to $8.6 \mathrm{keV}$ are given

\begin{tabular}{llll}
\hline & $\begin{array}{l}\sigma\left(\Delta Q^{2}\right) / \Gamma_{\gamma \gamma} \\
(\mathrm{pb} / \mathrm{keV}) \\
\text { LUMI }\end{array}$ & $\begin{array}{l}\sigma\left(\Delta Q^{2}\right) / \Gamma_{\gamma \gamma} \\
(\mathrm{pb} / \mathrm{keV})\end{array}$ & Probability $(\%)$ \\
VSAT & \\
\hline Data & $1.3 \pm 0.5$ & $5 \pm 5$ & \\
MC $\rho$-pole & 0.051 & 0.81 & $4.3(4.0-4.7)$ \\
MC J-pole & 0.85 & 2.1 & $58(34-81)$ \\
MC flat & 1.6 & 2.3 & $70(82-30)$ \\
\hline
\end{tabular}




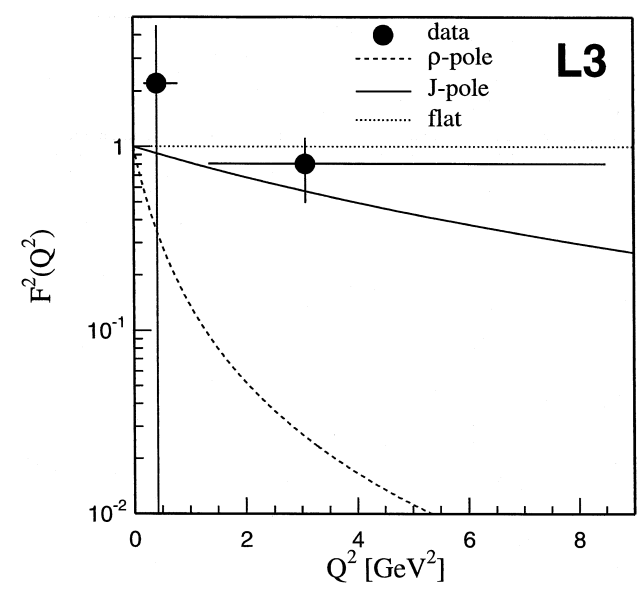

Fig. 5. The form factor $F^{2}\left(Q^{2}\right)$ vs. $Q^{2}$. The points represent the tagged data, while the three curves describe the form factor shape for a $\rho$-pole, J-pole or flat Monte Carlo.

of $\Gamma_{\gamma \gamma}$ within its measured error $( \pm 1.7 \mathrm{keV})$ are given in parentheses in the last column. The J-pole form factor and the flat form factor are clearly favoured over the $\rho$-pole form factor.

Since $\sigma\left(\Delta Q^{2}\right)$ is proportional to $F^{2}\left(Q^{2}\right)$, the latter can be obtained by dividing the measured cross section (normalised to the measured two-photon width) by the Monte Carlo cross section with a flat form factor, for which $F^{2}=1$,

$F^{2}\left(Q^{2}\right)=\frac{\sigma_{\text {data }}\left(\Delta Q^{2}\right)}{\sigma_{\text {flat MC }}\left(\Delta Q^{2}\right)}$.

The $F^{2}\left(Q^{2}\right)$ measured in the two $Q^{2}$ intervals is shown in Fig. 5, together with three curves corresponding to the theoretical predictions for a $\rho$-pole, J-pole and flat form factor. For a J-pole Monte Carlo, the average $Q^{2}$ for events with a tag in the LUMI is $3.08 \mathrm{GeV}^{2}$, and for events with a tag in the VSAT it is $0.42 \mathrm{GeV}^{2}$. If the data are fitted with Eq. (4) with the pole mass $\Lambda$ left free, we obtain $\Lambda=$ $5.3_{-2.5}^{+\infty} \mathrm{GeV}$. The $95 \%$ confidence level lower limit on the pole mass is $1.6 \mathrm{GeV}$.

\section{Conclusions}

The charmonium resonance $\eta_{c}$ is observed through the reconstruction of nine different decay modes at $\sqrt{s}=91 \mathrm{GeV}$ and at $\sqrt{s}=183 \mathrm{GeV}$. The two-photon width is determined to be $\Gamma_{\gamma \gamma}\left(\eta_{c}\right)=6.9 \pm 1.7$ stat \pm $0.8 \mathrm{sys} \pm 2.0$ (BR) $\mathrm{keV}$, in agreement with earlier measurements. This corresponds to $76 \pm 19$ signal events in total. A $\chi_{\mathrm{c} 0}$ signal of $29 \pm 11$ events is also observed, but $\Gamma_{\gamma \gamma}\left(\chi_{\mathrm{c} 0}\right)$ cannot be evaluated since its branching ratios are unknown. We find upper limits $\Gamma_{\gamma \gamma}\left(\chi_{\mathrm{c} 0}\right)<5.5 \mathrm{keV}, \Gamma_{\gamma \gamma}\left(\chi_{\mathrm{c} 2}\right)<1.4$ $\mathrm{keV}$, and $\Gamma_{\gamma \gamma}\left(\eta_{c}^{\prime}\right)<2.0 \mathrm{keV}$. Using tagged $\eta_{c}$ events we establish that the $Q^{2}$ dependence of the cross section is better described by a J-mass pole in the Vector Meson Dominance form factor than by a $\rho$-mass pole.

\section{Acknowledgements}

We wish to express our gratitude to the CERN accelerator division for the excellent performance of the LEP machine. We acknowledge the contributions of the engineers and technicians who have participated in the construction and maintenance of this experiment.

\section{References}

[1] V.M. Budnev et al., Phys. Rep. 15 (1974) 181.

[2] L.D. Landau, Dokl. Akad. Nauk. USSR 60 (1948) 207; C.N. Yang, Phys. Rev. 77 (1950) 242.

[3] Crystal Ball Collaboration, C. Edwards et al., Phys. Rev. Lett. 48 (1982) 70.

[4] Delphi Collaboration, P. Abreu et al., Phys. Lett. B 441 (1998) 479.

[5] E.S. Ackleh, T. Barnes, Phys. Rev. D 45 (1992) 232; T. Barnes, in: Proc. the IXth workshop on photon-photon collisions, World Scientific, 1992, p. 263; M.R. Ahmady, R.R. Mendel, Phys. Rev. D 51 (1995) 141; C.R. Münz, Nucl. Phys. A 609 (1996) 364; H.-W. Huang et al., Phys. Rev. D 56 (1997) 368.

[6] Th. Feldmann, P. Kroll, Phys. Lett. B 413 (1997) 410.

[7] G.A. Schuler, F.A. Berends, R. van Gulik, Nucl. Phys. B 523 (1998) 423

[8] L3 Collaboration, O. Adriani et al., Phys. Lett. B 318 (1993) 575.

[9] Particle Data Group, C. Caso et al., Eur. Phys. J. C 3 (1998) 1.

[10] L3 Collaboration, B. Adeva et al., Nucl. Instr. Meth. A 289 (1990) 35; M. Chemarin et al., Nucl. Instr. Meth. A 349 
(1994) 345; M. Acciarri et al., Nucl. Instr. Meth. A 351 (1994) 300; G. Basti et al., Nucl. Instr. Meth. A 374 (1996) 293; I.C. Brock et al., Nucl. Instr. Meth. A 381 (1996) 236; A. Adam et al., Nucl. Instr. Meth. A 383 (1996) 342.

[11] T. van Rhee, Ph.D. thesis in preparation, University of Utrecht.

[12] P. Béné et al., Nucl. Instr. Meth. A 306 (1991) 150.

[13] F.L. Linde, Ph.D. thesis, University of Leiden, 1988, unpublished.

[14] The L3 detector simulation is based on GEANT version 3.15. See R. Brun et al., GEANT 3, CERN DD/EE/84-1 (Revised), September 1987. The GHEISHA program (H. Fesefeldt, RWTH Aachen Report PITHA 85/02 (1985)) is used to simulate hadronic interactions.

[15] Crystal Ball Collaboration, Gaiser et al., Phys. Rev. D 34 (1986) 711.

[16] PLUTO Collaboration, Ch. Berger et al., Phys. Lett. B 167 (1986) 120; TPC $/ 2 \gamma$ Collab., H. Aihara et al., Phys. Rev. Lett. 60 (1988) 2355; CLEO Collaboration, W.-Y. Chen et al., Phys. Lett. B 243 (1990) 169; ARGUS Collaboration, H. Albrecht et al., Phys. Lett. B 338 (1994) 390; E760 Collaboration, T.A. Armstrong et al., Phys. Rev. D 52 (1995) 4839.

[17] CLEO Collaboration, Chen et al., Phys. Lett. B 243 (1990) 169; R.A. Lee, Crystal Ball Collaboration, Ph.D. thesis, SLAC-Report- 282 (1985).

[18] L3 Collaboration, M. Acciarri et al., Phys. Lett. B 453 (1999) 73.

[19] K.T. Chao, Y.F. Gu, S.F. Tuan, On Trigluonia in Charmonium Physics, BIhep-th/-93-45, PUTP-93-24, and UH-511790-94, 1993; S.F. Tuan, Hadronic Decay Puzzle in Charmonium Physics, UH-511-812-94 (1994), and Proceedings of the 6th Annual Hadron Spectroscopy and Structure Colloqium (HSSC94), Collega Park, MD, USA, 1994.

[20] TPC $/ 2 \gamma$ Collab., H. Aihara et al., Phys. Rev. Lett. 64 (1990) 172; CELLO Collaboration, H.-J. Berends et al., Z. Phys. C 49 (1991) 401; L3 Collaboration, M. Acciarri et al., Phys. Lett. B 418 (1998) 399; CLEO Collaboration, J. Gronberg et al., Phys. Rev. D 57 (1998) 33. 